\title{
Diaphragmatic paralysis following blunt neck trauma
}

\author{
Hesham Rashad Omar • Engy Helal • \\ Devanand Mangar · Enrico Mario Camporesi
}

Received: 3 December 2012/ Accepted: 23 January 2013/Published online: 12 February 2013

(C) SIMI 2013

A 25 -year-old construction worker presented to the
emergency department (ED) after the accidental fall of a
metal bar on his neck. In the field, he was appropriately
managed with a cervical collar for possible spine injury.
On arrival at the ED, he was fully conscious with severe
neck pain, hypotensive $80 / 50 \mathrm{mmHg}$ and bradycardiac
48 beats/min. Physical examination revealed a flaccid
paralysis and sensory loss in all four extremities. A chest
X-ray study was unremarkable (Fig. 1, panel a). Two
hours later, after examination revealed absent air entry

H. R. Omar $(\bowtie)$

Internal Medicine Department,

Mercy Hospital and Medical Center,

2525 South Michigan Avenue,

Chicago, IL 60616, USA

e-mail: hesham_omar2003@yahoo.com

E. Helal

Emergency Department,

Alagouza Police Hospital,

Cairo, Egypt

D. Mangar

Chief of Anesthesiology and CEO,

Florida Gulf to Bay Anesthesia,

Tampa General Hospital, TAMPA,

Florida, USA

\section{E. M. Camporesi}

Professor of Surgery/Anesthesiology,

Professor of Molecular Pharmacology and Physiology,

University of South Florida,

Tampa, FL, USA over the right lung base, a repeat X-ray study showed an elevated right hemidiaphragm and an ipsilateral mediastinal shift (Fig. 1 panel b). Computed tomography (CT scan) of the neck showed a comminuted, posteriorly displaced fracture of the third cervical vertebrae (Fig. 1, panel $\mathrm{c}$ and $\mathrm{d}$ ). In view of the established quadriplegia and neurogenic shock, this X-ray finding is suggestive of unilateral diaphragmatic paralysis. The delayed onset of diaphragmatic paralysis probably coincides with the evolving post-traumatic spinal cord edema. A few hours later, he developed respiratory distress with evident abdominal wall retraction during inspiration and protrusion during expiration in addition to hypoxia, hypercapnia and respiratory acidosis on blood gas analysis. The trachea was intubated and mechanical ventilation was started. This worsening respiratory status was concerning for the progression into bilateral diaphragmatic paralysis. Throughout the ICU course, his neurological status failed to improve requiring continued mechanical ventilation and subsequent tracheostomy. He was transferred to a subacute rehabilitation facility for further care.

The diagnosis of diaphragmatic paralysis is suggested clinically by the presence of abdominal wall retraction during inspiration and protrusion during expiration. Whereas unilateral diaphragmatic paralysis is usually well tolerated in the absence of underlying lung pathology, bilateral paralysis requires long-term ventilatory support. In contrast to bilateral diaphragmatic paralysis, a chest X-ray study is more specific for diagnosing a unilateral paralysis. Fluoroscopy confirms the diagnosis when paradoxical inspiratory elevation of the paralyzed hemidiaphragm is evident.

Conflict of interest None. 

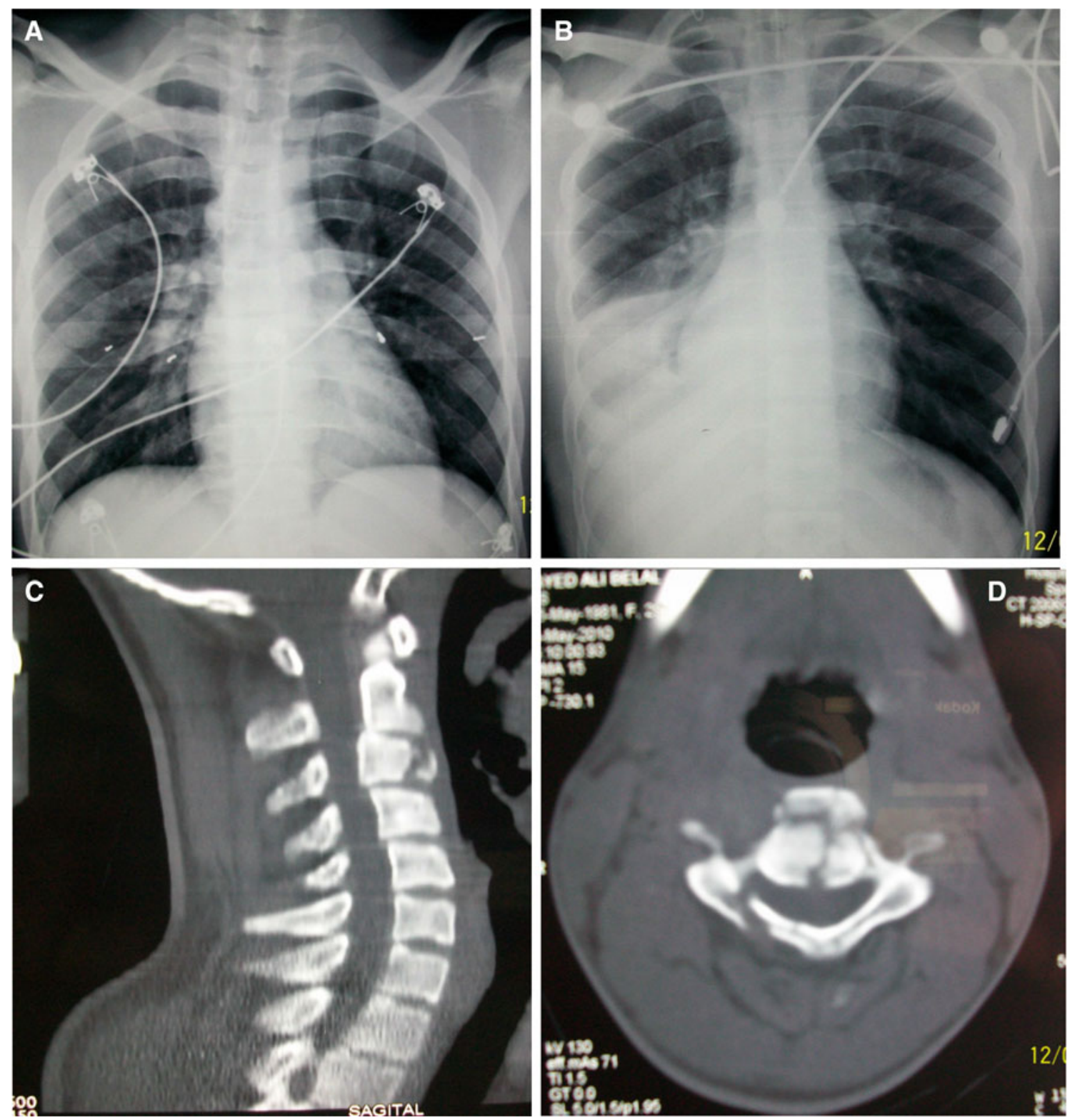

Fig. 1 a Normal chest X-ray. b chest X-ray performed $2 \mathrm{~h}$ later revealing significant elevation of the right hemidiaphragm up to the right infrahilar region with ipsilateral cardiomediastinal shift. CT scan of the neck in the sagittal (c) and axial (d) plane showing an acute

comminuted fracture of $\mathrm{C} 3$ vertebral body with slight posterior displacement of the major posterior fragment into the spinal canal causing probable mass effect upon the traversing spinal cord 